\title{
Ressources génétiques animales et logiques sociales : le maintien d'une race rustique en Savoie, la brebis Thônes et Marthod
}

\author{
Jacques Godet*, Thierry Joly \\ Institut supérieur d'agriculture Rhône-Alpes (Isara), \\ 31, Place Bellecour, 69288 Lyon cedex 02, France
}

\begin{abstract}
Social network and genetic resources: the 'Thônes et Marthod' sheep husbandry in Savoia. The management of local genetic resources implies the development of the possibilities. This requires that the producers be involved. However, they are not a homogeneous group and their goals vary greatly. The Thones and Marthod sheep husbandry, in Savoia (a French alpine area), is a good example. This hairy breed of sheep is very useful for range management, but its carcass has no value. Its survival can be explained by the local economy, as two-thirds of the Savoian peasants are part-time farmers. Such a pluriactivity allows these smallholders to keep a few ewes as currency. As well, some breeders rear flocks for commercial crossing and others have started dairy farming. This diversity has led the local association of sheep owners to consider the best way to organise and exchange. Thus, an adaptive, participatory research was launched to provide them with useful data. The demographic study of this endangered breed was completed by a sociological survey, as it is essential to understand the criteria and practices of the various producers. Although acting in distinctive ways, these farmers form a network that could be developed. Provided they would not be compelled to integrate a pyramidal system, they would be interested in improving ram exchanges through a kind of stud. It would be a very open system, each stock farmer will get the opportunity to become a breeder. It does not aim at a selection scheme but rather a social, organisational process which would allow the breeders to adapt the management of the breed in a sustainable way. Thus, the fact that these breeders reject integration does not necessarily mean they do not approve of partnership. (c) Inra/Elsevier, Paris
\end{abstract}

endangered breed / ovine / farming systems / ram breeding / genetic resources management / social research

Résumé - La gestion in situ des ressources génétiques dépend des possibilités de valorisation. Elle implique une concertation avec les éleveurs. Or, c'est un milieu

* Correspondance et tirés à part 
souvent hétérogène, aux objectifs diversifiés. Le cas de la brebis Thônes et Marthod, en Savoie, résume bien ces contraintes. Cette race rustique valorise l'espace mais un manque de conformation restreint ses débouchés. Son maintien est lié à la pluriactivité, qui permet à de petites structures de conserver quelques bêtes en appoint, pour un marché de proximité, bien qu'il y ait de gros élevages orientés vers le croisement industriel et des installations en production laitière. Cette diversité amène l'association des éleveurs à réfléchir sur les formes possibles d'organisation et à améliorer sa communication tant interne qu'externe. Une recherche pluridisciplinaire a complété l'étude démographique de la race par une enquête sociologique : typologie des éleveurs et analyse de leurs logiques de fonctionnement. Le schéma des échanges de reproducteurs laisse entrevoir une maîtrise possible de la voie mâle. D'où le projet d'un parc à béliers qui permette à tout éleveur de devenir fournisseur sans pour autant qu'on lui impose de rotation obligatoire. Il s'agit de favoriser un fonctionnement naturel en réseau, à l'exclusion de toute forme d'intégration. (c) Inra/Elsevier, Paris

ovin / population à petit effectif / gestion in situ / typologie des éleveurs / approche sociologique

\section{INTRODUCTION}

Le maintien de la diversité génétique par maintien des races à petits effectifs implique une gestion concertée avec les éleveurs. Ce choix politique est motivé par des considérations économiques et sociales en plus des motifs patrimoniaux et environnementaux. L'évolution vers la recherche d'une agriculture durable, la valorisation de la ressource paysagère et les attentes qualitatives du consommateur redonnent de l'intérêt à l'aspect proprement génétique et aux orientations de sélection des races à petits effectifs. Cela pose toutefois le problème de l'actualisation des systèmes de production et de l'application d'un programme de gestion génétique compatible avec leurs contraintes de fonctionnement.

La sensibilisation des éleveurs ne signifie pas pour autant convergence de leurs intérêts dans un contexte où les enjeux sont inégaux, les moyens réduits et les débouchés incertains. L'information sur les races, les élevages et les éleveurs eux-mêmes, reste à préciser. Et quand bien même les objectifs de production seraient clairement identifiés, toute une organisation est à mettre au point qui surmonte contraintes et disparités dans un milieu hétérogène qui ne regroupe pas que des professionnels. Si elle s'appuie sur une dynamique agricole, elle ne se limite pas à cette seule dimension et nécessite un partenariat élargi.

C'est dans cette perspective que s'inscrit ce travail de recherche, entrepris en Savoie avec des éleveurs ovins concernés au premier chef par une telle problématique. À quelles conditions est-il pensable de développer la race locale? Quelle serait la forme d'organisation la plus pertinente? Quelles préconisations techniques pour la gestion génétique de cette population sont susceptibles d'être appropriées par les éleveurs?

\section{MATÉRIEL ET MÉTHODES}

Notre choix s'est porté sur une race locale, la Thônes et Marthod, une race à petits effectifs qui résume l'essentiel des enjeux en matière de conservation. La Thônes et Marthod, élevée principalement dans les deux départements de Savoie et Haute-Savoie, a pris son nom en 1955, suite à la fusion de deux 
populations voisines : la Marthod ou mauriennaise, proche des races alpines d'Italie, et la Thônes, en partie dérivée du Nez Noir du Valais. C'est un animal rustique, aux aptitudes de marche à hautes altitudes reconnues, et capable de s'adapter à un régime «accordéon» sévère. Cependant, son point faible réside dans sa conformation bouchère où les carcasses sont essentiellement classées en $\mathrm{O}$ ou $\mathrm{P}$. La plupart des éleveurs sont adhérents à l'union des éleveurs de Thônes et Marthod, une association de sauvegarde qui définit ses objectifs indépendamment d'organismes spécialisés. La relance d'un programme de conservation en 1992 a permis d'initier un certain nombre d'actions, ce qui contribue au rapprochement des acteurs : marquage des béliers, gestion de primes, mise en relation de vendeurs et d'acheteurs.

Cette étude repose sur une double approche. La caractérisation des élevages consiste aussi bien à décrire la population ovine qu'à saisir la logique de fonctionnement des éleveurs et mieux les identifier. L'étude démographique des effectifs ovins s'est vue complétée par l'interview de près de $3 / 4$ des éleveurs de Thônes et Marthod, ce qui représente un atout méthodologique pour étudier cette population à petits effectifs.

L'enquête démographique s'est faite à l'échelle du troupeau et à l'échelle des reproducteurs en suivant une approche transversale et longitudinale ( $\mathrm{Vu}$ Thien Khang, 1983). L'absence d'identification des brebis et de carnets d'agnelage rendait impossible une étude génétique précise. L'objectif a donc été un bilan à dires d'éleveurs combinés à l'observation in situ d'une partie des élevages. Le recueil de témoignages d'une cinquantaine d'éleveurs a permis de réaliser, d'une part, l'inventaire et la description des élevages et d'autre part, l'historique des échanges de reproducteurs entre élevages. L'enquête sociologique, basée sur l'entretien semi-directif, a ciblé un échantillon plus réduit, d'une trentaine de personnes, mais incluant des éleveurs non encore visités. Cette approche qualitative se justifie à la fois par le matériau visé (les représentations autant que les faits) et par la non-pertinence d'une représentativité statistique. Les discours des éleveurs ont fait l'objet d'une analyse de contenu, recoupée par l'observation des pratiques et du système d'exploitation. L'objectif est de repérer les différentes catégories d'éleveurs et de comprendre leur logique de fonctionnement, y compris en s'intéressant aux déterminants non-professionnels.

Ces travaux ont fait l'objet d'une restitution aux éleveurs de Thônes et Marthod. Le but est d'entamer avec eux une recherche-action, qui catalyse leurs différentes attentes et leur permette de construire un outil en dialoguant mieux, entre eux comme avec leurs partenaires. Le principe est de leur renvoyer une vision globale de ce qu'ils sont ou pourraient être, de façon à ce que chacun dépasse la vision fragmentaire qu'il a des choses et se situe mieux par rapport à un projet commun. Il ne s'agit pas de casser ce fonctionnement en réseau - dont les études du Gerdal ${ }^{1}$ montrent combien il est opératoire -, mais au contraire de l'optimiser.

1 Gerdal : Groupe d'expérimentation recherche développement et actions localisées. Sociologie appliquée au développement rural. J-P. Darré et son équipe travaillent sur la production de connaissance dans les groupes professionnels locaux et sur les relations entre le discours scientifique et technique et les formes de connaissance des agriculteurs. Cf. Ci-après références bibliographiques. 


\section{RÉSULTATS}

\subsection{Situation démographique}

L'enquête a permis de repérer près de 80 élevages dont une soixantaine sont membres de l'association des éleveurs de Thônes et Marthod. Ces élevages sont de taille variable et pour plus de $90 \%$ localisés dans les pays savoyards.

Après une forte diminution des effectifs de brebis, passant de 32000 avantguerre à moins de 2500 en 1980, l'enquête porte le nombre d'animaux repérés à 3300 (Philit et al., 1996). L'âge moyen des brebis $(4,0$ ans) et le fort taux de renouvellement d'agnelles (25\%), en dépit d'une traditionnelle longévité de carrière (10\% ont plus de 10 ans), indique des installations ou des relances d'élevages récentes. La forte proportion de béliers (un pour 55 femelles) s'explique par l'éclatement des élevages en unités de taille variable et aux vocations de production différentes.

Les éleveurs de Thônes et Marthod sont jeunes avec une moyenne d'âge de 40 ans, mais présentent une grande diversité. L'étude révèle des objectifs de sélection divergents, des identités différentes et une attitude variable par rapport à la fonction de production. Bien que très importante, la dimension économique ne favorise pas pour autant la convergence des orientations et une logique de filière. La plupart de ces éleveurs sont des pluriactifs, soit parce qu'ils ne sont pas agriculteurs, soit parce qu'ils pratiquent une agriculture saisonnière ou diversifiée (agritourisme), soit encore parce qu'en tant qu'éleveurs, peu sont vraiment spécialisés ovins.

\subsection{Types d'élevages identifiés}

Un premier clivage s'observe entre la Maurienne et une région pour l'essentiel haut-savoyarde autour de Thônes, ce qui confirme, sur le plan zootechnique, la distinction que les éleveurs font du type mauriennais par rapport au standard général. Cette distinction reprend l'opposition des auteurs du XIX ${ }^{\mathrm{e}}$ siècle entre la Thônes élevée dans les vallées préalpines et la Marthod plus montagnarde conduite en alpage. Ces types confirment deux histoires différentes sur le plan socio-économique.

Pour la Maurienne, sa désindustrialisation la renvoie à une agriculture saisonnière et confirme sa vocation à l'élevage extensif. Le système d'élevage, basé sur des agnelages de printemps et la montée en estive, ne nécessite qu'une surveillance épisodique. L'éleveur de Thônes et Marthod a ici le visage d'un jeune de 35 ans, pratiquant le croisement industriel avec un troupeau de 150 brebis.

Cette situation contraste avec les petits élevages de la région de Thônes (entendue au sens large d'Annecy au Val d'Arly). Cette région est marquée par la proximité du tissu industriel et urbain, la faible altitude et l'étroitesse des structures. L'élevage ovin y représente une spéculation annexe, pour une population d'éleveurs âgée d'une cinquantaine d'années qui rassemble des pluriactifs, des retraités, des loueurs de gîte, des salariés travaillant en ville ou à l'usine... Cependant, quelle que soit la modicité du revenu occasionné, la plupart tirent parti économiquement de leur élevage, profitant d'un marché de proximité. Tous, enfin, obéissent à un réflexe identitaire très fort, «paysan» au 
sens propre du terme. La petite taille des troupeaux, pour la plupart conduits en race pure, ainsi que la disponibilité des éleveurs, autorisent l'agnelage d'automne.

Entre ces deux archétypes, élevage extensif de Maurienne et petit élevage des pluriactifs ou «urbains» du nord-Savoie, s'intercalent d'autres logiques plus spécialisées tant sur le plan géographique qu'en tant que systèmes. D’un côté, quelques gros élevages viande confrontés aux limites des filières classiques décapitalisent leur cheptel. De l'autre, des élevages laitiers se développent dans une nouvelle voie qui retrouve la vocation fromagère de cette race rustique, tout en jouant de sa dimension patrimoniale pour exploiter le créneau «vente fermière». Les éleveurs laitiers visent l'étalement des mises-bas sur l'année. Enfin, quelques élevages extérieurs à la région sont apparemment très divers (exploitations, élevages de loisirs, fermes pédagogiques,...) mais restent à mieux identifier.

Comme toute typologie, celle-ci est schématique. Mais par-delà les spécificités savoyardes et l'importance de la pluriactivité, elle n'est pas sans confirmer d'autres études (Audiot et al., 1983; Flamant et al., 1989; Quéméré, 1993).

\subsection{Le rejet d'une intégration}

Les débats au sein de l'Union des éleveurs de Thônes et Marthod sont de bons indicateurs des disparités et surtout des tensions résultant d'une situation socio-économique instable.

Les discussions sur le standard reflètent plus qu'une diversité régionale. Aux rapports de force classiques entre les sélectionneurs, soucieux de contrôler l'offre, et les utilisateurs plus pragmatiques, s'ajoute la montée de nouvelles logiques, pas nécessairement convergentes, et qu'incarnent les éleveurs laitiers ou les jeunes agriculteurs de Maurienne. Par leur côté professionnel, ces débats peuvent indisposer ceux qui s'intéressent à cette race précisément en tant que valeur ou activité marginale.

Un débat tout aussi révélateur porte sur l'aide de la Communauté européenne aux élevages en race pure de plus de vingt têtes. Plus que ses effets réels comme octroi de moyen, les mesures agri-environnementales ont révélé ostensiblement les clivages et ont été ressenties comme un instrument de sélection et de marginalisation, reléguant les petits élevages dans l'amateurisme.

Derrière ces tensions, se cache le problème d'enjeux économiques et sociaux différemment perçus. Un grand nombre d'éleveurs ne sont pas dans une logique de production spécialisée, voire même dans une logique de production stricto sensu, la Thônes et Marthod symbolisant avant tout une part d'identité irréductible. Il est révélateur que le tondeur soit le seul partenaire «extérieur» spontanément évoqué dans les interviews. Cela ne signifie nullement qu'il n'y ait pas une logique économique dans l'attitude des éleveurs, mais on doit tenir compte de deux facteurs. D'une part, leur attachement à la race symbolise la résistance des éleveurs à une forme de développement et d'encadrement professionnel. D'autre part, le système repose sur une adaptation très souple au contexte socio-économique local basé sur la vente directe à une clientèle de proximité. L'efficacité de ce système a longtemps résidé dans le fait qu'il est précisément marginal et fondé sur un réseau parallèle plutôt que sur une organisation formalisée. 
Dans les Alpes du Nord, l'élevage du mouton a toujours joué traditionnellement un rôle d'appoint, de trésorerie, de valeur refuge aux côtés de l'élevage bovin et des ressources du colportage et autres métiers saisonniers. Mais cet élevage, dans son ensemble, ne relevait pas d'une logique professionnelle.

\section{DISCUSSION}

\subsection{De quelles marges de manœuvre dispose-t-on pour gérer la diversité génétique?}

La tenue du carnet d'agnelage est incomplète, les contrôles de performance sont très peu pratiqués et le nombre de fournisseurs est réduit. Cependant, les trois principaux offreurs de mâles ne sont pas apparentés entre eux, les cas d'utilisation prolongée de béliers restent limités et la pratique de l'allottement est suffisamment maîtrisée pour atténuer les risques d'une augmentation rapide de la consanguinité de cette race.

Cependant, même si la situation de la Thônes et Marthod ne semble pas catastrophique, il est urgent d'accroître le nombre d'éleveurs fournisseurs de reproducteurs. Les trois principaux fournisseurs sont représentatifs de la typologie d'ensemble : l'un est spécialisé lait, l'autre axé sur la production de carcasses, le troisième un pluriactif attaché au phénotype traditionnel du pays de Thônes. Ces deux derniers élevages décapitalisent, ce qui peut être une chance de positionnement pour de petits élevages qui perdurent du fait d'un marché de proximité autant que d'une passion d'amateurs. Le système n'a donc pas trop souffert, jusqu'à présent, du rétrécissement des débouchés industriels mais cette situation reste aléatoire, car les normes communautaires en matière d'abattage risquent d'affecter ce marché de proximité. Les petits élevages représentent un vivier de fournisseurs potentiel important à valoriser. Mais ceux-ci sont-ils prêts à endosser ce rôle? Quels sont les relais à assurer et à mettre en œuvre?

La coexistence même de plusieurs options zootechniques permet de maintenir la diversité mais avive le problème d'une gestion globale de cette population à petits effectifs.

Le système agraire d'avant-guerre, tout autant que l'économie marginale permise ensuite en Savoie par la pluriactivité, favorisaient la non-spécialisation de la race. À l'encontre de la politique de «fixation des gènes» occasionnée par la modernisation de l'élevage, ces économies domestiques ont fait persister un certain polymorphisme des populations locales. Qu'en est-il maintenant? Jusqu'à quel point peut-on actualiser cette ressource «traditionnelle»? Quels sont les déterminants socio-économiques qui pèsent à terme dans la définition des objectifs de sélection?

La question n'est pas que des intérêts forcément divers deviennent vraiment communs, mais qu'ils puissent s'articuler comme complémentarités. Pour les producteurs de viande, la crise actuelle oblige à optimiser les performances de production, la rusticité de la Thônes ne suffisant pas à entraîner des économies d'échelle. Les éleveurs laitiers cherchent à redévelopper le potentiel de la race sur ce plan. Parallèlement, ce contexte peut détourner les purs amateurs des finalités économiques au profit d'une collection de phénotypes idéaux. Enfin, la 
valorisation de l'élevage comme activité de service réhabilite l'exploitant comme «entrepreneur » passant contrat avec une collectivité, la Thônes jouant déjà les débroussailleuses dans le cadre d'un accord avec le Parc Régional de Chartreuse. Le défi qui se pose à l'Union est donc, pour les prochaines années et sur la base d'un effectif réduit et dispersé, d'anticiper et de gérer cette diversification.

\subsection{Une affaire de communication pour l'Union des éleveurs}

Cette situation est précisément l'occasion d'enclencher une dynamique de concertation. L'idée d'un parc à béliers situé en alpage a permis d'initier des discussions où les risques sanitaires et les problèmes pratiques (éloignement, coût de l'opération, agnelages tardifs...) ont été invoqués. Cependant, le fond du débat n'est pas dans un problème d'information technique; les éleveurs expriment leurs craintes d'une imposition, d'une logique collective anticipant les choix à faire sur le plan économique et social. L'exemple du mouton Boulonnais, sauvé in extremis, montre que, de pierre d'achoppement, le parc à béliers peut devenir un point de ralliement, un élément fédérateur, pour peu qu'on respecte certaines règles de communication. Par communication, on entend ici autant un comportement social qu'une diffusion d'information.

Pour le Boulonnais, un schéma de rotation des familles avait d'abord été envisagé (Stievenard, 1993). Ce schéma a été abandonné pour un système plus simple autorisant tout éleveur à devenir fournisseur, alors qu'auparavant les reproducteurs provenaient de trois élevages sur-représentés. En 1986, l'Union des éleveurs de Thônes et Marthod a elle aussi tiré les enseignements du rejet d'un schéma collectif basé sur une rotation obligatoire des béliers (Rochambeau, 1983), schéma alors préconisé par le Groupement régional des producteurs ovins. Le principe est de préserver des marges de manœuvre et de rassurer en évitant tout excès de programmation et d'encadrement. Il s'agit autant de donner à tout éleveur l'occasion de devenir un fournisseur de génétique que de lui laisser le libre choix du reproducteur, quels que soient les conseils donnés et la sélection d'améliorateurs proposée par les gestionnaires du parc.

L'objectif est bien de faire du parc à béliers un cadre d'échanges, et d'abord au sens social du terme, un lieu de rencontres et un outil de communication. Cette fonction pédagogique, en jouant la participation plutôt que l'imposition, devrait permettre au parc de remplir au plus vite son rôle technique et informatif. Sur ce plan, c'est aussi se donner la chance d'une construction en commun, progressive et négociée, des objectifs de sélection. Par «sélection», on entend ici le compromis à trouver pour actualiser un système domestique sans pour autant intégrer un programme de sélection stricto sensu, défini comme «forme industrielle» (Casabianca et Vallerand, 1994). Toute période de changement accroît le souci de l'éleveur de contrôler les incertitudes. L'innovation génétique doit faire l'objet d'un apprentissage de groupe, d'une réappropriation paritaire, au risque d'être perçue comme un transfert d'autorité.

Cela renforce l'Union dans son rôle de médiateur et la nécessité qu'il y a de travailler sa communication tant interne qu'externe. L'enjeu est important car il s'agit de l'activation d'un réseau en tant que tel, et non pas d'un problème institutionnel de vulgarisation. La mobilisation de l'éleveur n'est pas affaire de compréhension littérale du message technique. Il raisonne à partir de sa situation générale, de l'ensemble de ses contraintes et d'une appréciation des 
risques à ce niveau. Le technicien ou le scientifique raisonnent la préservation du patrimoine génétique à l'échelle de la population sans intégrer ces déterminants socio-économiques que beaucoup résument par des idéologies ou comme des mentalités. Comme le rappelle Darré, «les gens usent des mots pour dire non les choses mais leur relation aux choses» (Darré, 1985). Il y a variation des sens selon les groupes sociaux. C'est d'autant plus vrai dans le cas des éleveurs de Thônes et Marthod, qu'ils ne forment pas vraiment un groupe professionnel local au sens ou l'entend Darré.

\section{CONCLUSIONS, PERSPECTIVES}

Rentrer dans les systèmes de pensée des éleveurs n'est pas affaire de philosophie, mais le préalable à des applications concrètes. D'une façon générale, ces observations confirment qu'on ne peut raisonner la gestion des ressources génétiques indépendamment des facteurs structurels et conjoncturels qui conditionnent les logiques sociales d'un territoire donné. La stratégie de l'Union vise à renforcer le sentiment de parité entre éleveurs et élargir son audience sans imposer de relation pyramidale. Le développement de partenariats ponctuels avec des interlocuteurs scientifiques et techniques, est partie intégrante de cette stratégie de concertation. La conception de schémas de gestion génétique adaptés à cette dynamique locale, dans le cadre de réseaux de dialogue, est un défi stimulant pour les généticiens. Cependant, elle implique au préalable, la reconnaissance d'une culture technique locale, d'une forme pré-existante de gestion, et l'acceptation d'une logique de négociation et de traduction mutuelle des représentations. C'est à ces conditions que peut s'élaborer une culture de transition qui préserve les éleveurs dans leur identité sociale, celle des premiers gestionnaires des ressources génétiques animales, tout en favorisant une représentation globale, incarnée, concrètement, par cet outil d'apprentissage en commun qu'est le parc à béliers.

\section{REMERCIEMENTS}

Ces enquêtes menées en Savoie et Haute-Savoie durant l'été 1996 ont fait l'objet d'un mémoire de fin d'études (La Thônes et Marthod : historique, caractéristiques zootechniques et étude démographique) soutenu par M. Philit $\left(24^{\mathrm{e}}\right.$ promotion) sous la responsabilité de T. Joly, et d'un rapport d'étude (Thônes et Marthod : une race à faible effectif, les conditions économiques et sociales de son maintien) par un groupe d'élèves-ingénieurs de $4^{\mathrm{e}}$ année (P. Bernard, B. Chareyron, C. Gamon et L. Gisquet), sous la responsabilité de J. Godet. L'investissement des étudiants sur le terrain a été facilité par l'accueil cordial et motivé des éleveurs de Thônes et Marthod.

Les auteurs sont redevables à Clovis Chatel, secrétaire de l'Union des éleveurs, de l'appui précieux qu'il a apporté à cette étude et à Annick Audiot, de l'Inra, de ses remarques et critiques attentives, qui ont largement contribué à la rédaction de cet article.

Cette étude a été réalisée grâce au soutien financier du ministère de l'enseignement supérieur et de la recherche (Décision d'aide $\mathrm{n}^{\circ} 95 \mathrm{G}$ 0092). 


\section{RÉFÉRENCES}

Audiot A., Gibon A., Flamant J.C., La conservation des races menacées : quels éleveurs?, Ethnozootechnie 33 (1983) 77-81.

Casabianca F., Vallerand F., Gérer les races locales d'animaux domestiques : une dialectique entre ressources génétiques et développement régional, Gen. Sel. Evol. 26 (1) (1994) 343-367.

Darré J.P., La parole et la technique : l'univers de pensée des éleveurs du Ternois, L'Harmattan, Paris, 1985.

Darré J.P., Pairs et experts dans l'agriculture : dialogues et production de connaissance pour l'action. Erès, Ramonville St-Agne (1994).

Flamant J.C., Cabannes-Audiot A., Vallerand F., Les populations humaines gestionnaires des populations animales, in : Brossier J., Valceschini E. (éd.), Les exploitations agricoles et leur environnement, essais sur l'espace technique et économique, colloque de La Bussière Inra, 143-160, 1989.

Philit M., Chatel C., Godet J., Joly T., Étude démographique d'une race ovine à petits effectifs : la Thônes et Marthod, Renc. Rech. Ruminants 3 (1996) 327.

Quéméré $P$., Peut-il y avoir conservation d'une race en péril sans valorisation économique? Éléments de réponse dans le cas de la race bovine Bretonne Pie-Noire, Ethnozootechnie 52 (1993) 25-32.

Rochambeau H. de, Méthode de gestion des petites populations, Ethnozootechnie 33 (1983) 55-61.

Stievenard R., Comment créer ou encadrer une dynamique des éleveurs? Ethnozootechnie 52 (1993) 55-68.

$\mathrm{Vu}$ Thien Khang J., Méthodes d'analyse des données démographiques et généalogiques dans les populations d'animaux domestiques, Gen. Sel. Evol. 15 (1983) 263-298. 